\title{
Bogoliubov theory of entanglement in a Bose-Einstein condensate
}

\author{
Anders Søndberg Sørensen \\ Institute of Physics and Astronomy, University of Aarhus, \\ DK-8000 Aarhus C, Denmark
}

\begin{abstract}
We consider a Bose-Einstein condensate which is illuminated by a short resonant light pulse that coherently couples two internal states of the atoms. We show that the subsequent time evolution prepares the atoms in an interesting entangled state called a spin squeezed state. This evolution is analysed in detail by developing a Bogoliubov theory which describes the entanglement of the atoms. Our calculation is a consistent expansion in $1 / \sqrt{N}$, where $N$ is the number of particles in the condensate, and our theory predict that it is possible to produce spin squeezing by at least a factor of $1 / \sqrt{N}$. Within the Bogoliubov approximation this result is independent of temperature.
\end{abstract}

PACS numbers: 03.65.Ud, 03.75.Fi, 42.50.Dv

\section{INTRODUCTION}

One of the main problems in the experimental explorations of entanglement is the engineering of a controlled interaction between individual quantum particles. Such an interaction among the particles is automatically present in Bose-Einstein condensed dilute atomic gases [1, 2. 3], where the experimental results show clear signatures of the collisions of the particles. It is therefore natural to try to exploit this interaction to create entanglement and a number of proposals have appeared which use the collisional interaction to entangle the atoms in the condensate [1, 5, 6, 月, 8, 9, 10, 11].

In Ref. 低 we proposed a realistic experiment where the atoms in a Bose-Einstein condensate are illuminated by a single resonant pulse. After this pulse the system evolves freely, and the collisional interaction among the particles prepares the atoms in an entangled state. The created entangled state even has practical applications in atomic clocks, where it can be used to increase the precision significantly [12]. In [4] we presented an approximate simulation of the condensate dynamics, where the state vector was expanded on subspaces containing different number of atoms, and where the spatial wavefunction in each subspace was evolved with the GrossPitaevskii equation. This calculation indicated that a substantial entanglement can be created, but the validity of the ansatz used in the calculation and the behaviour of the proposal at non-zero temperature is difficult to determine from the simulation. An exact calculation with a stochastic method [8] has later confirmed that the proposal is indeed capable of producing substantial entanglement. But due to the noise in this calculation, it is difficult to estimate the exact amount of entanglement from this procedure and the method is also difficult to apply in the regime where most experiments are currently operating.

In this paper we investigate the proposal [4 in more detail. We first develop a Bogoliubov theory for a two component condensate and then we apply it to describe the entanglement of the atoms in the condensate. Our theory is a consistent expansion in the ratio between non-condensed and condensed particles and the validity of the approximation can directly be investigated for a given experimental configuration. To lowest order, the Bogoliubov theory can be used to describe the effect of a non-vanishing temperature, and our theory indicates that within the Bogoliubov approximation the created entanglement is independent of temperature. The results of the Bogoliubov theory agrees very well with the results of the approximate method used in Ref. [4] and thus confirms the validity of this method.

\section{A. Considered experimental setup}

We consider a collection of $N$ atoms with two internal states $a$ and $b$. We assume that we first create a condensate consisting only of atoms in the internal state $a$. After the preparation of the condensate a fast resonant pulse is applied to the atoms. The pulse is assumed to be much faster than any other time scale in the problem, so that the only action of the pulse is to mix the internal states $|a\rangle \rightarrow \cos (\phi / 2)|a\rangle+\sin (\phi / 2)|b\rangle$ and $|b\rangle \rightarrow \cos (\phi / 2)|b\rangle-\sin (\phi / 2)|a\rangle$. The theory developed in section III and IV applies for all values of the angle $\phi$ but we only go into details with the case where the resonant interaction is a $\pi / 2$ pulse, $\phi=\pi / 2$. In this paper we shall describe our atoms in the second quantization using field operators $\hat{\Psi}_{a}$ and $\hat{\Psi}_{b}$, and we calculate the time evolution in the Heisenberg picture, where the action of the pulse is given by

$$
\begin{aligned}
\hat{\Psi}_{a}\left(\vec{r}, t=0^{+}\right)= & \cos \left(\frac{\phi}{2}\right) \hat{\Psi}_{a}\left(\vec{r}, t=0^{-}\right) \\
& -\sin \left(\frac{\phi}{2}\right) \hat{\Psi}_{b}\left(\vec{r}, t=0^{-}\right) \\
\hat{\Psi}_{b}\left(\vec{r}, t=0^{+}\right)= & \cos \left(\frac{\phi}{2}\right) \hat{\Psi}_{b}\left(\vec{r}, t=0^{-}\right) \\
& +\sin \left(\frac{\phi}{2}\right) \hat{\Psi}_{a}\left(\vec{r}, t=0^{-}\right)
\end{aligned}
$$

( $t=0^{-}$and $t=0^{+}$denotes the time just before and after the pulse). After the pulse we let the system evolve 
and perform a measurement on it at a later time.

The resonant pulse splits the condensate into an $a$ and a $b$ component with a well defined phase between them; if one applies a new pulse shortly after the first, it is possible to produce interferences between the two component and, e.g., transfer all the atoms back into the $a$ state. As the two components evolve with time there is nothing to maintain the relative phase between them, and the relative phase will spread in time because each of the two components do not have a well defined number of particles 13, 14, 15]. Due to the spreading of the relative phase, the magnitude of interference terms like $\left\langle\hat{\Psi}_{a}^{\dagger} \hat{\Psi}_{b}\right\rangle$ will decrease with time and it will no longer be possible to see interferences between the two components. This process is referred to as phase collapse.

Instead of focusing on the interferences between the condensates it is fruitful to consider other observables of the system. By considering fluctuations in the particle numbers one can show that the time evolution prepares the atoms in an entangled state called a spin squeezed stated.

\section{B. Spin squeezing}

Suppose that we prepare $N$ atoms in an equal superposition of two internal state $(|a\rangle+|b\rangle) / \sqrt{2}$ and measure the number of particles in the $a$ state. Because the particles are independent of each other the result of the measurement will be a distributed according to the binomial distribution with a mean value $\bar{N}_{a}=N / 2$ and a variance $N / 4$. A spin squeezed state is a state where the atoms are prepared in a suitable entangled state such that the variance (or noise) of this measurement is reduced.

A more convenient way to represent the atoms is to consider each atom as a spin- $1 / 2$ particle with the internal states $|a\rangle$ and $|b\rangle$ representing the spin up and spin down states respectively. In this language the noise in the counting statistics is represented by the variance of the $J_{z}$ operator $\left(\Delta J_{z}\right)^{2}$, where the collective $J_{x}, J_{y}$, and $J_{z}$ operators are obtained by summing the spin operators for the individual atoms. Expressed in terms of the spin operators, spin squeezing is the reduction of the noise of $J_{z}$ (or any other spin component). Recently the first experimental realizations of such spin squeezed states have been achieved 16, 17, 18, 19.

In Ramsey spectroscopy, as used for instance in atomic clocks, a signal is recorded which is proportional to the length of the spin in a given direction, say the $x$ direction, and the noise of the signal is proportional to the noise of a spin component $J_{\theta}=\cos (\theta) J_{z}+\sin (\theta) J_{y}$ perpendicular to the mean spin. The precision of atomic clocks is currently limited by the spin noise $\left(\Delta J_{\theta}\right)^{2}$ [20], and by using a spin squeezed state where this noise is smaller than in the unsqueezed states, which are used today, it is possible to increase the precision of the clock. Wineland et al. [12] have analysed this possibility and have shown that the frequency variance can be reduced by a factor of

$$
\xi_{\theta}^{2}=\frac{N\left(\Delta J_{\theta}\right)^{2}}{\left\langle J_{x}\right\rangle^{2}}
$$

by preparing the atoms in a spin squeezed state with $\xi_{\theta}^{2}<1$. This points to an interesting application of spin squeezed states in atomic clocks.

It has also been shown that a reduction of the squeezing parameter below unity $\xi_{\theta}^{2}<1$ requires the atoms to be in an entangled state [4], and to quantify the entanglement which may be obtained in the considered experimental setup, we shall determine the reduction of the squeezing parameter $\xi_{\theta}^{2}$. A different characterization of the squeezing is presented in Ref. [21], where the depth of entanglement in a collection of atoms is identified.

To see that the proposed experimental setup produces squeezing we shall first consider the short time evolution. At time $t=0^{+}$we have $\xi_{\theta}^{2}=1$ for all $\theta$, and to prove that squeezing is produced, it is sufficient to show that the time derivative of $\xi_{\theta}^{2}$ is negative for a suitable choice of $\theta$.

In the limit of very low temperatures a collection of atoms with two internal states $a$ and $b$ are described by the second quantized Hamiltonian

$$
\begin{aligned}
H= & \sum_{j=a, b} \int d^{3} r \hat{\Psi}_{j}^{\dagger}(\vec{r}) H_{0, j} \hat{\Psi}_{j}(\vec{r}) \\
& +\frac{1}{2} \sum_{j=a, b} g_{j j} \int d^{3} r \hat{\Psi}_{j}^{\dagger}(\vec{r}) \hat{\Psi}_{j}^{\dagger}(\vec{r}) \hat{\Psi}_{j}(\vec{r}) \hat{\Psi}_{j}(\vec{r}) \\
& +g_{a b} \int d^{3} r \hat{\Psi}_{a}^{\dagger}(\vec{r}) \hat{\Psi}_{b}^{\dagger}(\vec{r}) \hat{\Psi}_{a}(\vec{r}) \hat{\Psi}_{b}(\vec{r}),
\end{aligned}
$$

where $H_{0, j}$ is the one particle Hamiltonian for atoms in state $j$ including the kinetic energy and the external trapping potential $V_{j}(\vec{r})$, and $g_{j k}=4 \pi \hbar^{2} a_{s, j k} / M$ is the strength of the interaction between particles of type $j$ and $k$, expressed in terms of the scattering length $a_{s, j k}$ and the atomic mass $M$. Here we have assumed that there are no spin changing collisions. A specific experimental setup where it is possible to exclude such spin changing collision with sodium atoms in an optical trap is described in [4].

With the Hamiltonian (3) we may find the equation of motion for the field operator $\hat{\Psi}_{a}$ by taking the commutator with the Hamiltonian (using $\hbar=1$ )

$$
i \frac{d}{d t} \hat{\Psi}_{a}=H_{0, a} \hat{\Psi}_{a}+g_{a a} \hat{\Psi}_{a}^{\dagger} \hat{\Psi}_{a} \hat{\Psi}_{a}+g_{a b} \hat{\Psi}_{b}^{\dagger} \hat{\Psi}_{b} \hat{\Psi}_{a} .
$$

For brevity most of the equations in this papers will only be presented for the field operator for the $a$ component. The corresponding equations for the $b$ component can be achieved by exchanging subscripts $a$ and $b$. Since we work in the Heisenberg picture, essentially all operators and functions appearing in this paper will depend on time, and for brevity we shall in most cases omit the time argument on operators and functions. Unless specified 
otherwise operators and functions, like the field operators $\hat{\Psi}_{a}$ and $\hat{\Psi}_{b}$ in Eq. (田) should always be considered as functions of the time $t$. From the derivative in (位) we calculate the time derivative of the squeezing parameter $\xi_{\theta}^{2}$ at the time $t=0^{+}$, and by using the relation (11) with $\phi=\pi / 2$ we can express the derivative in terms of the field operators before the pulse

$$
\begin{aligned}
\frac{d}{d t} \xi_{\theta}^{2}= & \sin (2 \theta) \frac{g_{a a}+g_{b b}-2 g_{a b}}{2 N} \times \\
& \int d^{3} r\left\langle\hat{\Psi}_{a}^{\dagger}\left(\vec{r}, t=0^{-}\right)^{2} \hat{\Psi}_{a}\left(\vec{r}, t=0^{-}\right)^{2}\right\rangle .
\end{aligned}
$$

If $g_{a a}+g_{b b} \neq 2 g_{a b}$ we can always choose the angle $\theta$ such that the expression on the right hand side is negative and this exact equation immediately proves that squeezing will be produced. The time derivative is proportional to the two point correlation function which in the limit of an ideal condensate is $N(N-1) \int d^{3} r|\Phi|^{4}$, where $\Phi$ is the condensate wavefunction before the pulse.

The above argument shows that squeezing will be produced but it does not quantify the amount of squeezing which is produced. A simple estimate of the squeezing can be obtained by making a single mode approximation to each of the components. In this approximation the part of the Hamiltonian (3) which is responsible for spin squeezing can be written as [4, [7, 8]

$$
H_{\text {spin }}=\chi J_{z}^{2}
$$

with the coupling constant $\chi$ given by

$$
\chi(t)=\int d^{3} r \frac{g_{a a}\left|\Phi_{a}^{\mathrm{ex}}\right|^{4}+g_{b b}\left|\Phi_{b}^{\mathrm{ex}}\right|^{4}-2 g_{a b}\left|\Phi_{a}^{\mathrm{ex}}\right|^{2}\left|\Phi_{b}^{\mathrm{ex}}\right|^{2}}{2},
$$

where $\Phi_{a}^{\mathrm{ex}}\left(\Phi_{b}^{\mathrm{ex}}\right)$ is the condensate wavefunction for particles of type $a(b)$ (the "ex" is used to indicate the exact wavefunction because it will later be important to distinguish it from low order approximations to it).

The squeezing arising from the Hamiltonian (6) can be solved analytically [22]. The obtainable squeezing is approximately $\xi_{\theta}^{2} \approx N^{-2 / 3}$, and this indicate that strong squeezing can be produced if the condensate contains a large number of atoms. The validity of the arguments leading to the Hamiltonian (6) are not quite clear from the derivation, and it is the main purpose of this paper to investigate the validity of this simple description. As we shall see below, the Hamiltonian (6) is indeed a good approximation to the dynamics of the system but the coupling constant (7) has to be modified slightly. To obtain this result we describe the system by Bogoliubov theory.

\section{Bogoliubov theory}

The Hamiltonian (3) and the resulting Eq. (4) are far to complicated to be solved in general and approximations have to be applied. Here we shall determine the time evolution from the Hamiltonian (3) by applying the Bogoliubov approximation. The standard formulation of the Bogoliubov approximation assumes a broken symmetry such that the field operators have a non-vanishing mean value $\left\langle\hat{\Psi}_{a}(\vec{r}, t)\right\rangle=\sqrt{\bar{N}_{a}} \Phi_{a}^{\mathrm{ex}}(\vec{r}, t)$, where $\bar{N}_{a}$ is the mean number of particles of type $a$. The Bogoliubov method proceeds by splitting the field operators according to

$$
\begin{aligned}
& \hat{\Psi}_{a}(\vec{r}, t)=\sqrt{N_{a}} \Phi_{a}^{\mathrm{ex}}(\vec{r}, t)+\delta \hat{\Psi}_{a}(\vec{r}, t) \\
& \hat{\Psi}_{b}(\vec{r}, t)=\sqrt{N_{b}} \Phi_{b}^{\mathrm{ex}}(\vec{r}, t)+\delta \hat{\Psi}_{b}(\vec{r}, t) .
\end{aligned}
$$

The idea behind this splitting is that the fluctuations $\delta \hat{\Psi}_{a}$ and $\delta \hat{\Psi}_{b}$ are much smaller than the contribution of the condensates, and simple equations for the field operators are obtained by expanding the Hamiltonian (3) to low order in the $\delta \hat{\Psi}$ s.

The splitting (8) gives some nice properties, such as simple commutation relations for the $\delta \hat{\Psi}$ operators, but unfortunately it is not well suited for our present purpose. As we shall see below, the phase collapse and entanglement mainly arise from the evolution of the atoms in the condensate modes. In Eq. (8) the quantum fluctuations of the condensate modes and fluctuations perpendicular to these modes are treated equally, and this is not a good approximation to the system. In a direct numerical integration we have found that the $\delta \hat{\Psi}_{\mathrm{s}}$ grow very quickly due to the dynamics in the condensate modes so that the assumptions of the theory break down after a short period of time 23.

To circumvent the problems associated with the splitting (8) we employ a different splitting which enables a better description of the evolution in the condensate modes

$$
\begin{aligned}
& \hat{\Psi}_{a}(\vec{r}, t)=\hat{a} \Phi_{a}^{\operatorname{ex}}(\vec{r}, t)+\delta \hat{\Psi}_{a}(\vec{r}, t) \\
& \hat{\Psi}_{b}(\vec{r}, t)=\hat{b} \Phi_{b}^{\operatorname{ex}}(\vec{r}, t)+\delta \hat{\Psi}_{b}(\vec{r}, t),
\end{aligned}
$$

where the operator $\hat{a}$ and $\hat{b}$ obey bosonic commutation relations $\left[\hat{a}, \hat{a}^{\dagger}\right]=1$ and $[\hat{a}, \hat{b}]=0$. In this paper we shall derive the time evolution of the field operators by assuming that the fluctuations $\delta \hat{\Psi}_{a}$ and $\delta \hat{\Psi}_{b}$ are much smaller than the contributions from the condensate modes. An approach similar to ours has previously been considered in Refs. 115, 24, 25 where particle number conserving Bogoliubov approximations are derived. We shall generalize these previous results to a situation where the atoms can be in two different internal states and where the number of atoms in each of the states may not be well defined. This generalization enables us to describe the collapse of the relative phase between the two condensates and the entanglement of the atoms, which are shown to arise from the time evolution of the operators $\hat{a}$ and $\hat{b}$.

In section II we present the assumptions used in the Bogoliubov description [15, 24 and in section III we derive the equations of motion for the relevant operators. In 
section [V] we solve these equation and in $\square$ we apply our theory to the considered experimental setup described in subsection I A. Finally, we end the paper with a conclusion in section V1.

\section{ASSUMPTIONS}

In this section we describe the assumptions of our theory, which is presented in sections III and IV. The assumptions and methods used here are very similar to the two theories derived for a one component condensate by Castin and Dum [15] and by Gardiner [24]. These two derivations are equivalent but use two different approaches. Castin and Dum derive equations of motion for the relevant field operators and these equations are approximated by inserting a splitting as in Eq. (9) and neglecting high order terms in $\delta \hat{\Psi}$. In the work of Gardiner the approximations are made to the Hamiltonian, where terms of high order in $\delta \hat{\Psi}$ are neglected before the equations of motion are derived. Here we choose to follow the derivation and notation of Castin and Dum in Ref. [15] very closely. In order to make the paper self contained we reproduce most of the calculations of Castin and Dum.

We assume that the atoms are cooled to a very low temperature such that a condensate is formed before the pulse which mixes the two internal states. The condensate wavefunction is determined from the one-body density operator which is given by

$$
\left\langle\vec{r}^{\prime}\left|\rho_{a, 1}\right| \vec{r}\right\rangle=\left\langle\hat{\Psi}_{a}^{\dagger}(\vec{r}) \hat{\Psi}_{a}\left(\vec{r}^{\prime}\right)\right\rangle .
$$

Mathematically, the definition of a condensate is that $\rho_{a, 1}$ has an eigenvalue

$$
\rho_{a, 1}\left|\Phi_{a}^{\mathrm{ex}}\right\rangle=N_{a}^{\mathrm{ex}}\left|\Phi_{a}^{\mathrm{ex}}\right\rangle,
$$

which is much larger than all other eigenvalues $\left(N_{a}^{\mathrm{ex}} \sim\right.$ $N$, where $N$ is the total number of atoms), and the spatial modefunction is given by $\Phi_{a}^{\mathrm{ex}}(\vec{r})$.

The resonant pulse mixes the field operators as described by Eq. (11), and by using that the initial state is the vacuum of $\Psi_{b}\left(t=0^{-}\right)$we find that $\rho_{a, 1}\left(t=0^{+}\right)=$ $\rho_{b, 1}\left(t=0^{+}\right)=\rho_{a, 1}\left(t=0^{-}\right) / 2$. After the pulse the state $\left|\Phi_{a}^{\mathrm{ex}}\left(t=0^{-}\right)\right\rangle$is still an eigenvalue of the density operator $\rho_{a, 1}$ with eigenvalue $N_{a}^{\operatorname{ex}}\left(t=0^{+}\right)=N_{a}^{\operatorname{ex}}\left(t=0^{-}\right) / 2$, so that we have a component of the condensate in both the $a$ and the $b$ state with wavefunctions

$$
\Phi_{a}^{\mathrm{ex}}\left(\vec{r}, t=0^{+}\right)=\Phi_{b}^{\mathrm{ex}}\left(\vec{r}, t=0^{+}\right)=\Phi_{a}^{\mathrm{ex}}\left(\vec{r}, t=0^{-}\right) .
$$

The condensate modefunctions $\Phi_{a}^{\mathrm{ex}}$ and $\Phi_{b}^{\mathrm{ex}}$ can be used to define a splitting of the field operator as in Eq. (9), and to describe the squeezing we need to determine the time evolution of $\delta \hat{\Psi}_{a}$ and $\hat{a}$. To derive the equations of motion for these operators it will be convenient to have an expression for them in terms of the field operator $\hat{\Psi}_{a}$. By normalizing $\left|\Phi_{a}^{\mathrm{ex}}\right\rangle$ to unity we obtain

$$
\hat{a}=\int d^{3} r \Phi_{a}^{\mathrm{ex} *}(\vec{r}) \hat{\Psi}_{a}(\vec{r})
$$

and

$$
\delta \hat{\Psi}_{a}(r)=\int d^{3} r^{\prime}\left\langle\vec{r}\left|Q_{a}^{\mathrm{ex}}\right| \vec{r}^{\prime}\right\rangle \hat{\Psi}_{a}\left(\vec{r}^{\prime}\right),
$$

where $Q_{a}^{\text {ex }}$ is the projector on the subspace perpendicular to $\left|\Phi_{a}^{\mathrm{ex}}\right\rangle: Q_{a}^{\mathrm{ex}}=1-\left|\Phi_{a}^{\mathrm{ex}}\right\rangle\left\langle\Phi_{a}^{\mathrm{ex}}\right|$. To simplify the notation we introduce the operand $\circ$ to denote the action of an operator $O$ onto a field operator such as $\hat{\Psi}_{a}$ :

$$
O \circ \hat{\Psi}_{a}=\int d^{3} r O|\vec{r}\rangle \hat{\Psi}_{a}(\vec{r})
$$

(o is also used in Ref. 115]). With this notation Eqs. (13) and (14) can be written in the simpler form

$$
\begin{aligned}
\hat{a} & =\left\langle\Phi_{a}^{\mathrm{ex}}\right| \circ \hat{\Psi}_{a} \\
\delta \hat{\Psi}_{a} & =Q_{a}^{\mathrm{ex}} \circ \hat{\Psi}_{a} .
\end{aligned}
$$

Our calculation assumes a condensate of weakly interacting atoms ( $a_{s}^{3} \rho \ll 1$, where $\rho$ is the atomic density) at very low temperature $T \approx 0$. In this limit the fraction of non-condensed atoms is very small, $\delta N / N \sim 10^{-2}-10^{-3}$ [26. The field operator $\hat{\Psi}_{a}$ has matrix elements scaling as $\sqrt{\bar{N}_{a}}$ from the condensed atoms and contributions of order $\sqrt{\delta N_{a}}$ from the non-condensed atoms, where $\bar{N}_{a}$ and $\delta N_{a}$ are the average number of particles and the number of uncondensed particles of type $a$. Due to the small ratio between non-condensed and condensed particles it is useful to perform an expansion of the field operator $\hat{\Psi}_{a}$ in terms of $\sqrt{\delta N_{a} / N_{a}}$. Formally this expansion is achieved by taking the limit

$$
\begin{aligned}
\bar{N}_{a} & \rightarrow \infty \\
\bar{N}_{a} g_{a a} & =\text { constant },
\end{aligned}
$$

while keeping a constant ratio between the scattering lengths and between the number of particles in the $a$ and the $b$ states. Below we show that $\delta N_{a}$ is of order unity in this limit, and our expansion in terms of $\sqrt{\delta N_{a} / \bar{N}_{a}}$ becomes an expansion in $1 / \sqrt{\bar{N}_{a}}$ (note that since we have a fixed ratio $\bar{N}_{a} / \bar{N}_{b}$ this also corresponds to an expansion in $1 / \sqrt{\bar{N}_{b}}$ ).

\section{EXPANSION IN POWERS OF $1 / \sqrt{\bar{N}_{a}}$}

In this section we present the explicit expansion in powers of $1 / \sqrt{\bar{N}_{a}}$. The expansion procedure is implemented by considering the time evolution of the operator 27

$$
\hat{\Lambda}_{a}^{\mathrm{ex}}(\vec{r}, t)=\frac{1}{{\sqrt{\bar{N}_{a}}}^{\dagger}} a^{\dagger}(t) \delta \hat{\Psi}_{a}(\vec{r}, t)
$$


From the definition of the condensate wavefunction (11) and the splitting (9) follows that the expectation value of $\hat{\Lambda}_{a}^{\mathrm{ex}}$ vanish exactly at all times

$$
\left\langle\hat{\Lambda}_{a}^{\mathrm{ex}}(\vec{r}, t)\right\rangle=0 .
$$

As we shall show below, $\hat{\Lambda}_{a}^{\mathrm{ex}}$ is of order unity in the limit in Eq. (17) so that the number of non-condensed atoms approaches a constant in this limit. To perform our expansion we write the operator $\hat{\Lambda}_{a}^{\mathrm{ex}}$ and the wavefunction as a series of terms

$$
\begin{aligned}
& \hat{\Lambda}_{a}^{\mathrm{ex}}=\hat{\Lambda}_{a}+\frac{1}{\sqrt{\bar{N}_{a}}} \hat{\Lambda}_{a}^{(1)}+\frac{1}{\bar{N}_{a}} \hat{\Lambda}_{a}^{(2)}+\ldots . \\
& \Phi_{a}^{\mathrm{ex}}=\Phi_{a}+\frac{1}{\sqrt{\bar{N}_{a}}} \Phi_{a}^{(1)}+\frac{1}{\bar{N}_{a}} \Phi_{a}^{(2)}+\ldots
\end{aligned}
$$

and we derive equations for each of the terms order by order. Since we require that the exact wavefunction is normalized to all orders in $1 / \sqrt{\bar{N}_{a}}$, the lowest order contribution must also be normalized $\left\langle\Phi_{a} \mid \Phi_{a}\right\rangle=1$.

The procedure in this section is to calculate $d \hat{\Lambda}_{a}^{\mathrm{ex}} / d t$ and $d \hat{a} / d t$ to order $k$ in $1 / \sqrt{\bar{N}_{a}}(k=-1,0)$ and take the mean value $\left\langle\hat{\Lambda}_{a}^{\mathrm{ex}}\right\rangle$ which must vanish to all orders in $1 / \sqrt{\bar{N}_{a}}$. With this procedure we calculate the contributions to the field operator to order $\bar{N}_{a}^{0}$. The main difference compared to the derivation of Castin and Dum in Ref. [15] is that in their derivation the number of atoms of type $a$ is fixed. This means that $\hat{N}_{0 a}-\bar{N}_{a}$, where $\hat{N}_{0 a}=\hat{a}^{\dagger} \hat{a}$ is the number operator for the condensed particles, is only of order $\bar{N}_{a}^{0} \sim 1$ because the difference only arises due to the non-condensed particles. In our situation the distribution on different number states is a binomial distribution with a width $\sim \sqrt{\bar{N}_{a}}$ and hence the operator $\hat{N}_{0 a}-\bar{N}_{a}$ is of order $\sqrt{\bar{N}_{a}}$.

\section{A. Basic equations for $\hat{\Lambda}_{a}^{\mathrm{ex}}$ and $\hat{a}$}

As a starting point for our calculation we use the time derivatives of the operators $\hat{\Lambda}_{a}^{\mathrm{ex}}$ and $\hat{a}$. By taking the time derivative of Eqs. (13) and (14) and using the definition of $\hat{\Lambda}_{a}^{\mathrm{ex}}$ (18) we obtain the expressions

$$
i \frac{d}{d t} \hat{a}=i\left[\left(\frac{d}{d t}\left\langle\Phi_{a}^{\mathrm{ex}}\right|\right) \circ \hat{\Psi}_{a}+\left\langle\Phi_{a}^{\mathrm{ex}}\right| \circ \frac{d}{d t} \hat{\Psi}_{a}\right]
$$

and

$$
\begin{aligned}
i \frac{d}{d t} \hat{\Lambda}_{a}^{\mathrm{ex}}= & \frac{i}{\sqrt{\bar{N}_{a}}}\left[\hat{a}^{\dagger} Q_{a}^{\mathrm{ex}} \circ\left(\frac{d}{d t} \hat{\Psi}_{a}-\hat{a} \frac{d}{d t}\left|\Phi_{a}^{\mathrm{ex}}\right\rangle\right)\right. \\
& +\left(\left(\frac{d}{d t} \hat{\Psi}_{a}^{\dagger}\right) \circ\left|\Phi_{a}^{\mathrm{ex}}\right\rangle+\hat{\Psi}_{a}^{\dagger} \circ \frac{d}{d t}\left|\Phi_{a}^{\mathrm{ex}}\right\rangle\right) \delta \hat{\Psi}_{a} \\
& \left.-\hat{a}^{\dagger}\left|\Phi_{a}^{\mathrm{ex}}\right\rangle\left(\frac{d}{d t}\left\langle\Phi_{a}^{\mathrm{ex}}\right|\right) \circ \delta \hat{\Psi}_{a}\right]
\end{aligned}
$$

In these expressions the only time derivatives are of the wavefunction $\Phi_{a}^{\text {ex }}$ and the field operator $\hat{\Psi}_{a}$. By substituting the splitting of the field operators in Eq. (9) into the right hand side of the time derivative of $\hat{\Psi}_{a}$ in Eq. (4) and inserting this into the above expressions we may find equations of motion for $\hat{\Lambda}_{a}^{\mathrm{ex}}$ and $\hat{a}$ involving different powers of $\delta \hat{\Psi}_{a}$. Below we shall only consider the two lowest order contributions to $\hat{\Lambda}_{a}^{\mathrm{ex}}$ and $\hat{a}$, and it is sufficient to keep the terms which are linear in $\delta \hat{\Psi}_{a}$. Equivalently, this corresponds to neglecting the terms of higher than second order in the $\delta \hat{\Psi}$ operators in the Hamiltonian if we had used the approach of Gardiner [24], where the approximation are made to the Hamiltonian rather than in the equations of motion.

\section{B. Order $\sqrt{\bar{N}_{a}}$ : Gross-Pitaevskii equations}

The largest terms in the expression in Eq. (22) are the the terms on the first line. These two terms are of order $\sqrt{\bar{N}_{a}}$, but with a suitable definition of the wavefunction $\left|\Phi_{a}^{\mathrm{ex}}\right\rangle$ the two contributions cancel each other to leading order in $\sqrt{\bar{N}_{a}}$, and $\hat{\Lambda}_{a}^{\mathrm{ex}}$ is of order $\bar{N}_{a}^{0}$. By using Eqs. (4) and (9) and keeping only the dominant contribution from the condensed particles we get

$$
i \frac{d}{d t} \hat{\Lambda}_{a}^{\mathrm{ex}}=\sqrt{\bar{N}_{a}} Q_{a} \circ\left(H_{\mathrm{GP}, a}-i \frac{d}{d t}\right)\left|\Phi_{a}\right\rangle+O\left(\bar{N}_{a}^{0}\right),
$$

where we have introduced the Gross-Pitaevskii Hamiltonian

$$
H_{\mathrm{GP}, a}=H_{0, a}+g_{a a} \bar{N}_{a}\left|\Phi_{a}\right|^{2}+g_{a b} \bar{N}_{b}\left|\Phi_{b}\right|^{2} .
$$

In Eq. (23) we have replaced the number operators by their leading order contributions $\hat{a}^{\dagger} \hat{a} \approx \bar{N}_{a}$ and $\hat{a}^{\dagger} \hat{a}^{\dagger} \hat{a} \hat{a} \approx$ $\bar{N}_{a}^{2}$. The difference between the leading order and the operators is of higher order and will be taken into account below. From Eq. (23) follows that the $\sqrt{\bar{N}_{a}}$ contribution to $\hat{\Lambda}_{a}^{\mathrm{ex}}$ vanishes if we chose

$$
\left(-i \frac{d}{d t}+H_{\mathrm{GP}, a}\right)\left|\Phi_{a}\right\rangle=\zeta(t)\left|\Phi_{a}\right\rangle .
$$

The term on the right side introduces an optional phase factor on the wavefunction $\left|\Phi_{a}\right\rangle$ ( $\zeta$ must be real due to the normalization condition). Since all measurable quantities are expressed in terms of the field operator (9), we can introduce any phase factor on $\left|\Phi_{a}\right\rangle$ if we include the opposite phase factor on $\hat{a}$. Here shall choose the simplest possible phase evolution of $\hat{a}$ and we set $\zeta=0$ so that (25) reduces to the usual time dependent GrossPitaevskii equation. With this choice of $\zeta$ the equation of motion for $\hat{a}$ cancels to leading order $i d \hat{a} / d t=O\left(\bar{N}_{a}^{0}\right)$. 


\section{Order $\bar{N}_{a}^{0}: \Phi_{a}^{(1)}=0$.}

In this subsection we shall show that the lowest order correction to the wavefunction $\Phi_{a}^{(1)}$ vanishes exactly in the considered experimental situation. To show this, we consider the mean value of Eq. (22) to order $\bar{N}_{a}^{0}$

$$
\begin{aligned}
i \frac{d}{d t}\left\langle\hat{\Lambda}_{a}(\vec{r})\right\rangle= & \sqrt{\bar{N}_{a}}\langle\vec{r}| Q_{a}^{\mathrm{ex}} \circ\left(H_{0, a}+g_{a a} \bar{N}_{a}\left|\Phi_{a}^{\mathrm{ex}}\right|^{2}\right. \\
& \left.+g_{a b} \bar{N}_{b}\left|\Phi_{b}^{\mathrm{ex}}\right|^{2}-i \frac{d}{d t}\right)\left|\Phi_{a}^{\mathrm{ex}}\right\rangle+O\left(1 / \sqrt{\bar{N}_{a}}\right) .
\end{aligned}
$$

When calculating the mean value of the third term in Eq. (22) $\left\langle d \hat{\Psi}_{a}^{\dagger} / d t \delta \hat{\Psi}_{a}\right\rangle / \sqrt{\bar{N}_{a}}$ we need to consider mean values like $g_{a a}\left\langle\hat{a} \hat{a}^{\dagger} \hat{a}^{\dagger} \delta \hat{\Psi}_{a}\right\rangle / \sqrt{\bar{N}_{a}}$. These terms do not vanish and at first sight they might seem to contribute to the present order. But since $\left\langle\hat{\Lambda}_{a}^{\mathrm{ex}}\right\rangle$ vanishes, this term may be rewritten as $g_{a a}\left\langle\left(\hat{N}_{0 a}-\bar{N}_{a}\right) \hat{a}^{\dagger} \delta \hat{\Psi}_{a}\right\rangle / \sqrt{\bar{N}_{a}}$, and since the parenthesis is only of order $\sqrt{\bar{N}_{a}}$ this term does not contribute to the present order.

Because of the exact relation (19) the mean value of Eq. (22) must vanish to all orders in $\sqrt{\bar{N}_{a}}$. The $\sqrt{\bar{N}_{a}}$ contribution vanish due to the Gross-Pitaevskii equation (25). The next order is obtained by expanding $Q_{a}^{\mathrm{ex}}$ and $\Phi_{a}^{\mathrm{ex}}$ in Eq. (26) and we get

$$
\begin{aligned}
0=Q_{a} \circ & {\left[\left(-i \frac{d}{d t}+H_{0, a}\right) \Phi_{a}^{(1)}\right.} \\
& +g_{a a} \bar{N}_{a}\left(2\left|\Phi_{a}\right|^{2} \Phi_{a}^{(1)}+\Phi_{a}^{2} \Phi_{a}^{(1) *}\right) \\
& \left.+g_{a b} \bar{N}_{b}\left(\Phi_{a} \Phi_{b} \Phi_{b}^{(1)^{*}}+\Phi_{a} \Phi_{b}^{*} \Phi_{b}^{(1)}+\left|\Phi_{b}\right|^{2} \Phi_{a}^{(1)}\right)\right] .
\end{aligned}
$$

Due to the projection operator $Q_{a}$ this equation admits the inclusion of a an overall phase in $\Phi_{a}^{(1)}$, i.e., $i d \Phi_{a}^{(1)} / d t$ may have a contribution $\zeta^{(1)} \Phi_{a}$. Again we prefer to have the simplest possible equation for $\hat{a}$ and chose $\zeta^{(1)}=$ 0 . With this choice the equation for $\Phi_{a}^{(1)}$ is linear and homogeneous so that $\Phi_{a}^{(1)}$ vanishes if it vanishes at $t=$ $0^{+}$. In Ref. [15] it is shown that $\Phi_{a}^{(1)}=0$ in thermal equilibrium as we have just before the resonant pulse (11), and since the condensate wavefunction is exactly the same before and after the pulse, as described in Eq. (12), we have

$$
\Phi_{a}^{(1)}(t)=0
$$

for all $t$.

\section{Order $\bar{N}_{a}^{0}$ : Bogoliubov approximation}

With the result (28) we can now calculate the derivative in Eq. (21) to order $\bar{N}_{a}^{0}$ and we get

$$
\begin{aligned}
i \frac{d}{d t} \hat{a}= & g_{a a}\left\langle\left.\Phi_{a}|| \Phi_{a}\right|^{2} \mid \Phi_{a}\right\rangle\left(\hat{N}_{0 a}-\bar{N}_{a}\right) \hat{a} \\
& +g_{a b}\left\langle\left.\Phi_{a}|| \Phi_{b}\right|^{2} \mid \Phi_{a}\right\rangle\left(\hat{N}_{0 b}-\bar{N}_{b}\right) \hat{a} \\
& +g_{a a} \sqrt{\bar{N}_{a}}\left(\left\langle\left.\Phi_{a}|| \Phi_{a}\right|^{2} \circ \hat{\Lambda}_{a}+\hat{\Lambda}_{a}^{\dagger} \circ\left|\Phi_{a}\right|^{2} \mid \Phi_{a}\right\rangle\right) \hat{a} \\
& +g_{a b} \sqrt{\bar{N}_{b}}\left(\left\langle\Phi_{a}\left|\Phi_{b}^{*} \Phi_{a} \circ \hat{\Lambda}_{b}+\hat{\Lambda}_{b}^{\dagger} \circ \Phi_{a}^{*} \Phi_{b}\right| \Phi_{a}\right\rangle\right) \hat{a},
\end{aligned}
$$

where we have replaced expressions like $\hat{a}^{\dagger} \hat{a}^{\dagger} \hat{a} \hat{a}-\hat{a}^{\dagger} \hat{a} \bar{N}_{a}$ with their lowest order contributions $\left(\hat{N}_{0 a}-\bar{N}_{a}\right) \bar{N}_{a}$. The right hand side is seen to be a Hermitian operator multiplied by $\hat{a}$. This does not contribute to the time derivative of the number operator $\hat{N}_{0 a}$, so that we have

$$
\frac{d}{d t} \hat{N}_{0 a}=O\left(\bar{N}_{a}^{0}\right)
$$

This relation is consistent with $\hat{\Lambda}_{a}$ being of order unity. The number of particles of type $a$ is a conserved quantity and hence $\hat{N}_{0 a}+\delta \hat{\Psi}_{a}^{\dagger} \circ \delta \hat{\Psi}_{a}=\hat{N}_{0 a}+\hat{\Lambda}_{a}^{\dagger} \circ \hat{\Lambda}_{a}+O\left(1 / \sqrt{\bar{N}_{a}}\right)$ must be conserved. Since $\hat{\Lambda}_{a}$ is of order $\bar{N}_{a}^{0}$, we must have a relation like (30). The weak time dependence of the number of condensed particles indicate that essentially all particles stay in the condensate modes and this justifies the description of the system by single mode approximations. Note, however that such single mode calculations must be performed with care. A calculation which completely ignores the non-condensed particles only contains the first two lines in Eq. (29), but the remaining terms are of the same order as the first and should be kept in the calculation. As we shall see below these terms gives rise to the same interaction as in the single mode approximation but with a different coupling constant.

For the $\hat{\Lambda}$ s the equation of motion reads

$$
i \frac{d}{d t}\left[\begin{array}{c}
\hat{\Lambda}_{a} \\
\hat{\Lambda}_{b} \\
\hat{\Lambda}_{a}^{\dagger} \\
\hat{\Lambda}_{b}^{\dagger}
\end{array}\right]=\mathcal{L} \circ\left[\begin{array}{c}
\hat{\Lambda}_{a} \\
\hat{\Lambda}_{b} \\
\hat{\Lambda}_{a}^{\dagger} \\
\hat{\Lambda}_{b}^{\dagger}
\end{array}\right]+\left(\hat{N}_{0 a}-\bar{N}_{a}\right) \vec{\alpha}+\left(\hat{N}_{0 b}-\bar{N}_{b}\right) \vec{\beta},
$$

where the vectors $\vec{\alpha}$ and $\vec{\beta}$ are given by

$$
\vec{\alpha}=\left|\Phi_{a}\right|^{2}\left[\begin{array}{c}
g_{a a} \sqrt{\bar{N}_{a}}\left|\Phi_{a}\right\rangle \\
g_{a b} \sqrt{\bar{N}_{b}}\left|\Phi_{b}\right\rangle \\
-g_{a a} \sqrt{\bar{N}_{a}}\left|\Phi_{a}^{*}\right\rangle \\
-g_{a b} \sqrt{\bar{N}_{b}}\left|\Phi_{b}^{*}\right\rangle
\end{array}\right]
$$

and

$$
\vec{\beta}=\left|\Phi_{b}\right|^{2}\left[\begin{array}{c}
g_{a b} \sqrt{\bar{N}_{a}}\left|\Phi_{a}\right\rangle \\
g_{b b} \sqrt{\bar{N}_{b}}\left|\Phi_{b}\right\rangle \\
-g_{a b} \sqrt{\bar{N}_{a}}\left|\Phi_{a}^{*}\right\rangle \\
-g_{b b} \sqrt{\bar{N}_{b}}\left|\Phi_{b}^{*}\right\rangle
\end{array}\right]
$$


and where the matrix $\mathcal{L}$ is

$$
\left[\begin{array}{cccc}
H_{\mathrm{GP}, a}+Q_{a} g_{a a} \bar{N}_{a}\left|\Phi_{a}\right|^{2} Q_{a} & Q_{a} g_{a b} \sqrt{\bar{N}_{a} \bar{N}_{b}} \Phi_{a} \Phi_{b}^{*} Q_{b} & Q_{a} g_{a a} \bar{N}_{a} \Phi_{a}^{2} Q_{a}^{*} & Q_{a} g_{a b} \sqrt{\bar{N}_{a} \bar{N}_{b}} \Phi_{a} \Phi_{b} Q_{b}^{*} \\
Q_{b} g_{a b} \sqrt{\bar{N}_{a} \bar{N}_{b}} \Phi_{a}^{*} \Phi_{b} Q_{a} & H_{\mathrm{GP}, b}+Q_{b} g_{b b} \bar{N}_{b}\left|\Phi_{b}\right|^{2} Q_{b} & Q_{b} g_{a b} \sqrt{\bar{N}_{a} \bar{N}_{b}} \Phi_{a} \Phi_{b} Q_{a}^{*} & Q_{b} g_{b b} \bar{N}_{b} \Phi_{b}^{2} Q_{b}^{*} \\
-Q_{a}^{*} g_{a a} \bar{N}_{a} \Phi_{a}^{* 2} Q_{a} & -Q_{a}^{*} g_{a b} \sqrt{\bar{N}_{a} \bar{N}_{b}} \Phi_{a}^{*} \Phi_{b}^{*} Q_{b} & -H_{\mathrm{GP}, a}-Q_{a}^{*} g_{a a} \bar{N}_{a}\left|\Phi_{a}\right|^{2} Q_{a}^{*} & -Q_{a}^{*} g_{a b} \sqrt{\bar{N}_{a} \bar{N}_{b}} \Phi_{a}^{*} \Phi_{b} Q_{b}^{*} \\
-Q_{b}^{*} g_{a b} \sqrt{\bar{N}_{a} \bar{N}_{b}} \Phi_{a}^{*} \Phi_{b}^{*} Q_{a} & -Q_{b}^{*} g_{b b} \bar{N}_{b} \Phi_{b}^{* 2} Q_{b} & -Q_{a}^{*} g_{a b} \sqrt{\bar{N}_{a} \bar{N}_{b}} \Phi_{a} \Phi_{b}^{*} Q_{b}^{*} & -H_{\mathrm{GP}, b}-Q_{b}^{*} g_{b b} \bar{N}_{b}\left|\Phi_{b}\right|^{2} Q_{b}^{*}
\end{array}\right]
$$

Here we have introduced the projector $Q_{a}^{*}$ which projects onto the space orthogonal to $\left|\Phi_{a}^{*}\right\rangle: Q_{a}^{*}=1-\left|\Phi_{a}^{*}\right\rangle\left\langle\Phi_{a}^{*}\right|$ (note that $Q_{a}^{*} \neq Q_{a}^{\dagger}=Q_{a}$ ). The first part of Eq. (31) containing the operator $\mathcal{L}$ corresponds to the equation derived for a single component in Ref. [15. With a fixed number of particles in the $a$ and the $b$ states this is the only contribution to this order. In a situation where we cannot ignore the fluctuations in the particle numbers there are additional contributions due to the last two terms in Eq. (31). Physically, we can understand the origin of these terms by considering a subspace with a given number of particles $\mathcal{N}_{a}$ and $\mathcal{N}_{b}$ (of type $a$ and $b$ respectively). In this subspace the Gross-Pitaevskii equations are not the best approximations to the evolution of the condensate mode because they contain the average values $\bar{N}_{a}$ and $\bar{N}_{b}$ rather than $\mathcal{N}_{a}$ and $\mathcal{N}_{b}$. From the structure of the last two terms in Eq. (31) it is seen that these terms corrects for the "wrong" value of the particle numbers. The approximate method used in Refs. [4, 14 is designed capture this dependence on the particle numbers and in section $\mathrm{V}$ we show that the results obtained with the present method are very similar to the result

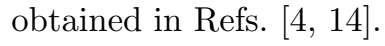

\section{SOLVING THE EQUATIONS}

With the equations (29) and (31) we have derived the equations of motion to the desired accuracy, and in this section we solve these equations. The solution is obtained by expanding the $\hat{\Lambda}$ operators on a suitable set of modefunctions at $t=0^{+}$. The time evolution of these modes is chosen such that the first term in Eq. (31) is automatically taken into account and we then treat the last two terms in Eq. 31. Finally the solution for the $\hat{\Lambda}$ operators is inserted into Eq. (29) which is then solved.

\section{A. Expansion at $t=0^{+}$}

Instead of having the operators $\hat{\Lambda}_{a}$ and $\hat{\Lambda}_{b}$ which have both a spatial part and an operator character, it is convenient to expand $\hat{\Lambda}_{a}$ and $\hat{\Lambda}_{b}$ on a set of vectors which take the spatial dependence into account. In such an ex- pansion, the expansion coefficients become operators and we have

$$
\left[\begin{array}{c}
\hat{\Lambda}_{a} \\
\hat{\Lambda}_{b} \\
\hat{\Lambda}_{a}^{\dagger} \\
\hat{\Lambda}_{b}^{\dagger}
\end{array}\right]=\sum_{k=1}^{\infty} \hat{c}_{k}\left[\begin{array}{c}
\left|u_{a k}\right\rangle \\
\left|u_{b k}\right\rangle \\
\left|v_{a k}\right\rangle \\
\left|v_{b k}\right\rangle
\end{array}\right]+\hat{c}_{k}^{\dagger}\left[\begin{array}{c}
\left|v_{a k}^{*}\right\rangle \\
\left|v_{b k}^{*}\right\rangle \\
\left|u_{a k}^{*}\right\rangle \\
\left|u_{b k}^{*}\right\rangle
\end{array}\right]
$$

The $u_{a}$ s and the $v_{a}$ s can be any functions perpendicular to $\Phi_{a}$ and $\Phi_{a}^{*}$ respectively, but the whole set must be chosen such that the entire space perpendicular to the condensates modes can be spanned by the vectors. A convenient choice of the functions is discussed in section V.

The $u$ and $v$ functions play the same role as the modefunction in ordinary Bogoliubov theory and it is an advantage to choose the standard normalization

$$
\begin{aligned}
\left\langle u_{a k} \mid u_{a k^{\prime}}\right\rangle+\left\langle u_{b k} \mid u_{b k^{\prime}}\right\rangle-\left\langle v_{a k} \mid v_{a k^{\prime}}\right\rangle-\left\langle v_{b k} \mid v_{b k^{\prime}}\right\rangle & =\delta_{k k^{\prime}} \\
\left\langle v_{a k}^{*} \mid u_{a k^{\prime}}\right\rangle+\left\langle v_{b k}^{*} \mid u_{b k^{\prime}}\right\rangle-\left\langle u_{a k}^{*} \mid v_{a k^{\prime}}\right\rangle-\left\langle u_{b k}^{*} \mid v_{b k^{\prime}}\right\rangle & =0 .
\end{aligned}
$$

With this normalization we may express the expansion operators $\hat{c}_{k}$ in terms of the $\hat{\Lambda}$ operators

$$
\hat{c}_{k}=\left\langle u_{a k}\right| \circ \hat{\Lambda}_{a}+\left\langle u_{b k}\right| \circ \hat{\Lambda}_{b}-\left\langle v_{a k}\right| \circ \hat{\Lambda}_{a}^{\dagger}-\left\langle v_{b k}\right| \circ \hat{\Lambda}_{b}^{\dagger},
$$

and from the commutation relation $\left[\hat{\Lambda}_{a}^{\dagger}, \hat{\Lambda}_{a}\right]=Q_{a}$ we find that the $\hat{c}_{k} \mathrm{~s}$ obey bosonic commutation relations $\left[\hat{c}_{k}, \hat{c}_{k^{\prime}}^{\dagger}\right]=\delta_{k k^{\prime}}$ and $\left[\hat{c}_{k}, \hat{c}_{k^{\prime}}\right]=0$.

\section{B. Time evolution}

To evolve the expansion (35) we need to choose the time evolution of the modefunctions. A convenient choice is

$$
i \frac{d}{d t}\left[\begin{array}{l}
\left|u_{a k}\right\rangle \\
\left|u_{b k}\right\rangle \\
\left|v_{a k}\right\rangle \\
\left|v_{b k}\right\rangle
\end{array}\right]=\mathcal{L} \circ\left[\begin{array}{c}
\left|u_{a k}\right\rangle \\
\left|u_{b k}\right\rangle \\
\left|v_{a k}\right\rangle \\
\left|v_{b k}\right\rangle
\end{array}\right]
$$

With this choice of time evolution, the normalization (36) is conserved and so is the commutation relation of the $\hat{c}_{k}$ 
operators. Indeed, the time evolution of $\hat{c}_{k}$ only comes from the fluctuations in the number of particles and is given by

$$
i \frac{d}{d t} \hat{c}_{k}=\left(\hat{N}_{0 a}-\bar{N}_{a}\right) f_{a k}+\left(\hat{N}_{0 b}-\bar{N}_{b}\right) f_{b k},
$$

where

$$
\begin{aligned}
f_{a k} & =\left(\left\langle u_{a k}\right|,\left\langle u_{b k}\right|,-\left\langle v_{a k}\right|,-\left\langle v_{b k}\right|\right) \cdot \vec{\alpha} \\
f_{b k} & =\left(\left\langle u_{a k}\right|,\left\langle u_{b k}\right|,-\left\langle v_{a k}\right|,-\left\langle v_{b k}\right|\right) \cdot \vec{\beta} .
\end{aligned}
$$

This equation is easily solved and by using that $\hat{N}_{0 a}$ and $\hat{N}_{0 b}$ do not depend on time to this order of approximation we find

$$
\begin{aligned}
\hat{c}_{k}(t)=\hat{c}_{k}\left(t=0^{+}\right) & -i\left(\hat{N}_{0 a}-\bar{N}_{a}\right) F_{a k}(t) \\
& -i\left(\hat{N}_{0 b}-\bar{N}_{b}\right) F_{b k}(t),
\end{aligned}
$$

where the functions $F_{a k}$ and $F_{b k}$ are defined by

$$
\begin{aligned}
& F_{a k}(t)=\int_{0}^{t} d t^{\prime} f_{a k}\left(t^{\prime}\right) \\
& F_{b k}(t)=\int_{0}^{t} d t^{\prime} f_{b k}\left(t^{\prime}\right) .
\end{aligned}
$$

Finally, by inserting Eqs. (35) and (41) into Eq. (29) we obtain

$$
\begin{aligned}
i \frac{d}{d t} \hat{a}= & \left(\hat{N}_{0 a}-\bar{N}_{a}\right)\left(g_{a a}\left\langle\left.\Phi_{a}|| \Phi_{a}\right|^{2} \mid \Phi_{a}\right\rangle+2 \sum_{k=1}^{\infty} \operatorname{Im}\left(f_{a k}^{*} F_{a k}\right)\right) \hat{a} \\
& +\left(\hat{N}_{0 b}-\bar{N}_{b}\right)\left(g_{a b}\left\langle\Phi_{a} \|\left.\Phi_{b}\right|^{2} \mid \Phi_{a}\right\rangle+2 \sum_{k=1}^{\infty} \operatorname{Im}\left(f_{a k}^{*} F_{b k}\right)\right) \hat{a}+\left(\sum_{k=1}^{\infty} f_{a k}^{*}(t) \hat{c}_{k}\left(t=0^{+}\right)+f_{a k}(t) \hat{c}_{k}^{\dagger}\left(t=0^{+}\right)\right) \hat{a} .
\end{aligned}
$$

The expression in Eq. (43) has the solution

$$
\hat{a}(t)=\exp \left[-i\left(\eta_{a}(t)+\hat{\Theta}_{a}(t)\right)\right] \hat{a}\left(t=0^{+}\right),
$$

where the Hermitian operator $\hat{\Theta}_{a}(t)$ is just the time integral from 0 to $t$ of the three terms multiplying $\hat{a}$ on right hand side of Eq. (43). The phase $\eta_{a}$ arises because the term involving the $\hat{c}_{k}$ and $\hat{c}_{k}^{\dagger}$ operators does not commute with itself at different times. By differentiating the expression in 44 with respect to time and using the Baker-Hausdorff relation a few times, we find that (44) is indeed a solution of (43) if the phase is given by

$$
\eta_{a}(t)=\sum_{k=1}^{\infty} \int_{0}^{t} d t^{\prime} \operatorname{Im}\left(F_{a k}\left(t^{\prime}\right) f_{a k}^{*}\left(t^{\prime}\right)\right) .
$$

A derivation of this phase and a geometrical interpretation of it can be found in [28]. With the expression (44) we have finished our derivation of the evolution of the field operator. In the next section we apply the developed theory to describe phase collapse and entanglement.

\section{APPLICATION OF THE THEORY}

In this section we apply the theory developed in sections III and IV to describe the collapse of the relative phase between the two condensates and the entanglement of the atoms. For simplicity we shall not be as general as in the previous sections and we only consider a symmetric interaction which we describe below.

\section{A. Symmetric interaction}

We consider the symmetric situation where $g_{a a}=$ $g_{b b} \neq g_{a b}$, and where the first pulse (1) is a $\pi / 2$ pulse such that $\bar{N}_{a}=\bar{N}_{b}$. We assume a system with a fixed total number of particles $N=2 \bar{N}_{a}$ so that we have

$$
\hat{N}_{0 a}-\bar{N}_{a}=-\left(\hat{N}_{0 b}-\bar{N}_{b}\right)=\left(\hat{N}_{0 a}-\hat{N}_{0 b}\right) / 2+O\left(\bar{N}_{a}^{0}\right) .
$$

We also assume that the trapping potentials for the two different internal states are identical, spherically symmetric, and harmonic $V_{a}=V_{b}=1 / 2 m \omega^{2} r^{2}$. With this symmetric choice of interactions the condensate wavefunctions are exactly the same for the two components of the condensate $\Phi_{a}=\Phi_{b}=\Phi$. Furthermore the symmetry can also be exploited in the Bogoliubov modes. We divide the sum over $k$ in Eq. (35) into a sum over terms which are even under the exchange of $a$ and $b$ ( + modes) and terms which are odd under exchange of $a$ and $b$ (- 
modes), i.e., we have

$$
\left[\begin{array}{l}
\left|u_{a k}\right\rangle \\
\left|u_{b k}\right\rangle \\
\left|v_{a k}\right\rangle \\
\left|v_{b k}\right\rangle
\end{array}\right]=\left[\begin{array}{l}
\left|u_{k}^{+}\right\rangle \\
\left|u_{k}^{+}\right\rangle \\
\left|v_{k}^{+}\right\rangle \\
\left|v_{k}^{+}\right\rangle
\end{array}\right] \text {or }\left[\begin{array}{c}
\left|u_{k}^{-}\right\rangle \\
-\left|u_{k}^{-}\right\rangle \\
\left|v_{k}^{-}\right\rangle \\
-\left|v_{k}^{-}\right\rangle
\end{array}\right] .
$$

In the remainder of this article superscript + and - on operators and functions will refer to these even and odd modes. The symmetry of the modes is reflected in the functions defined in section IV] which obey

$$
\begin{aligned}
f_{b k}^{ \pm} & = \pm f_{a k}^{ \pm} \\
F_{b k}^{ \pm} & = \pm F_{a k}^{ \pm} .
\end{aligned}
$$

From these relations we find that the two components have the same phase

$$
\eta_{a}=\eta_{b}
$$

and from the second relation and Eq. (46) follows that the operators describing the + modes are independent of time

$$
\frac{d}{d t} \hat{c}_{k}^{+}=0
$$

\section{B. Initial conditions}

To evaluate the time evolution of physical quantities we need to relate the different operators after the resonant pulse (1i) to the similar operators before the pulse. The relation between the operators before and after the pulse is completely describe by Eq. (1) and below we extract some result from the general relations.

Before the resonant pulse (1) we assume that a condensate is formed in the $a$ state and that there are no particles in the $b$ state. This means that $\hat{a}\left(t=0^{-}\right)$is of order $\sqrt{N}$ whereas $\hat{b}\left(t=0^{-}\right)$is of order unity so that we have from Eqs. (1) and (12) [29]

$$
\begin{aligned}
& \hat{a}\left(t=0^{+}\right)=\frac{1}{\sqrt{2}}\left(\hat{a}\left(0^{-}\right)-\hat{b}\left(0^{-}\right)\right) \approx \hat{a}\left(0^{-}\right) / \sqrt{2} \\
& \hat{b}\left(t=0^{+}\right)=\frac{1}{\sqrt{2}}\left(\hat{a}\left(0^{-}\right)+\hat{b}\left(0^{-}\right)\right) \approx \hat{a}\left(0^{-}\right) / \sqrt{2} .
\end{aligned}
$$

These approximations should be handled with care since they violate both the commutation relation and unitarity. The sign $\approx$ in Eq. (51) means that the operators have approximately the same matrix elements.

The operators $\hat{c}_{k}^{+}$can be related to the operators at $t=0^{-}$by using Eq. (37). Inserting the approximate relations in (51) we obtain

$$
\begin{aligned}
\hat{c}_{k}^{+}\left(t=0^{+}\right)=\sqrt{2} & \left(\left\langle u_{k}^{+}\left(0^{+}\right)\right| \circ \hat{\Lambda}_{a}\left(0^{-}\right)\right. \\
& \left.-\left\langle v_{k}^{+}\left(0^{+}\right)\right| \circ \hat{\Lambda}_{a}^{\dagger}\left(0^{-}\right)\right),
\end{aligned}
$$

where we have introduced a $\hat{\Lambda}$ operator before the $\pi / 2$ pulse $\hat{\Lambda}_{a}\left(0^{-}\right)=\hat{a}^{\dagger}\left(0^{-}\right) / \sqrt{N} \delta \hat{\Psi}_{a}\left(0^{-}\right)$which is also used in Ref. 15. As the other $\hat{\Lambda}$ operators, this operator can be expanded on a set of Bogoliubov modes $\hat{\Lambda}_{a}\left(0^{-}\right)=$ $\sum_{k} \hat{c}_{a k}\left(0^{-}\right)\left|u_{a k}\left(0^{-}\right)\right\rangle+\hat{c}_{a k}^{\dagger}\left(0^{-}\right)\left|v_{a k}\left(0^{-}\right)\right\rangle$, where the mode functions obey orthogonality relations similar to Eq. (36). In [15] it is shown that by a suitable choice of the modes $\left|u_{a k}\left(0^{-}\right)\right\rangle$and $\left|v_{a k}\left(0^{-}\right)\right\rangle$the pseudo-particle operators $\hat{c}_{a k}\left(0^{-}\right)$are the Bogoliubov operators describing excitations of the condensates, and the ground state is the vacuum of these operators. The simplest relation between the operators before and after the pulse is obtained by choosing $\left|u_{k}^{+}\left(0^{+}\right)\right\rangle=\left|u_{a k}\left(0^{-}\right)\right\rangle / \sqrt{2}$ and $\left|v_{k}^{+}\left(0^{+}\right)\right\rangle=\left|v_{a k}\left(0^{-}\right)\right\rangle / \sqrt{2}$. With these modes the operators describing quasi-particle excitations in the + modes are exactly the same as the operators describing excitations before the pulse

$$
\hat{c}_{k}^{+}\left(t=0^{+}\right)=\hat{c}_{a k}\left(t=0^{-}\right) .
$$

To find the initial condition for the operators $\hat{c}_{k}^{-}$we again use relation (37) and the approximation in Eq. (51) and we find [29]

$$
\begin{aligned}
\hat{c}_{k}^{-}\left(t=0^{+}\right)=-\frac{1}{\sqrt{\bar{N}_{a}}}( & \hat{a}^{\dagger}\left(0^{-}\right)\left\langle u_{k}^{-}\left(0^{+}\right)\right| \circ \delta \hat{\Psi}_{b}\left(0^{-}\right) \\
& \left.-\hat{a}\left(0^{-}\right)\left\langle v_{k}^{-}\left(0^{+}\right)\right| \circ \delta \hat{\Psi}_{b}^{\dagger}\left(0^{-}\right)\right) .
\end{aligned}
$$

The simplest initial condition is obtained by choosing $\left|v_{k}^{-}\left(0^{+}\right)\right\rangle=0$. With this choice $\hat{c}_{k}^{-}\left(0^{+}\right)$is proportional to $\delta \hat{\Psi}_{b}\left(0^{-}\right)$. Since the initial state is the vacuum of $\delta \hat{\Psi}_{b}\left(0^{-}\right)$ the initial state for $\hat{c}_{k}^{-}$is also the vacuum state independent of the modefunctions $\left|u_{k}^{-}\left(0^{+}\right)\right\rangle$and the state of the condensate. In our numerical work described below, we choose the $u_{k}^{-} \mathrm{s}$ to be the excited states of the GrossPitaevskii Hamiltonian before the pulse.

\section{Phase collapse}

We now investigate the collapse of the relative phase between the two components of the condensate. As a quantitative measure of the collapse we use the reduction of the mean spin in the $x$-direction which is defined by $\left\langle J_{x}\right\rangle=\operatorname{Re}\left(\left\langle J_{+}\right\rangle\right)$, where $J_{+}$is given in terms of the fields operators by $J_{+}=\hat{\Psi}_{a}^{\dagger} \circ \hat{\Psi}_{b}$. By inserting the splitting in Eq. (9), using the approximation in Eq. (51), and introducing the $\hat{\Lambda}$ s we obtain

$$
\left\langle J_{+}\right\rangle=\left\langle\hat{a}^{\dagger} \hat{b}\right\rangle+\left\langle\hat{\Lambda}_{a}^{\dagger} \circ \hat{\Lambda}_{b}\right\rangle+O\left(1 / \sqrt{\bar{N}_{a}}\right) .
$$

The first term in this expression represents the time evolution of the condensed atoms and the second term is the contribution from the non-condensed atoms. At short times the first term is of order $\bar{N}_{a}$ whereas the second 
term is of order unity. However, as we shall see below, the time scale of the collapse scales as $\sqrt{\bar{N}_{a}}$, and since $d \hat{\Lambda}_{a} / d t$ is of order unity, the second contribution may become comparable to the first at the collapse time. We shall only work in the dynamically stable region $g_{a b}<g_{a a}$. In the opposite case $g_{a b}>g_{a a}$ it is energetically favourable for the two components to separate so that any asymmetry in the two wavefunctions can grow with time and the system is dynamically unstable 14. By using the same techniques as was used to show a similar result in [15], one can show that equation of motion for the us and $v$ s (38) corresponds to the time evolution of a perturbation of the condensate wavefunction perpendicular to the wavefunction itself. Hence the stability of the system is determined by the stability of the solution of the GrossPitaevskii equation, and if the system is dynamically unstable the number of non-condensed particles is expected to grow exponentially with time, so that the approximations performed here are invalid. On the other hand, in the region $g_{a b}<g_{a a}$ it is energetically favourable for the two components to be overlapping so that the system is dynamically stable and the number of non-condensed particles performs small oscillations in time. This behaviour is confirmed by direct numerical integrations of the equations. In the stable region the contribution from non-condensed particles remain much smaller than the contribution of the condensed particles, and in the following we ignore the second term in Eq. (55).

When we insert the solution in Eq. (44) into Eq. (55) we see that the phase collapse arises from the difference of the angles $\hat{\Theta}_{a}-\hat{\Theta}_{b}$. By using the relations (46) and (48), the difference in the angles may be expressed as

$$
\hat{\Theta}_{a}-\hat{\Theta}_{b}=\left(\hat{N}_{0 a}-\hat{N}_{0 b}\right) \lambda+4 \operatorname{Re}\left(\sum_{k=1}^{\infty} F_{a k}^{-*} \hat{c}_{k}^{-}\left(t=0^{+}\right)\right),
$$

where the function $\lambda$ is determined by

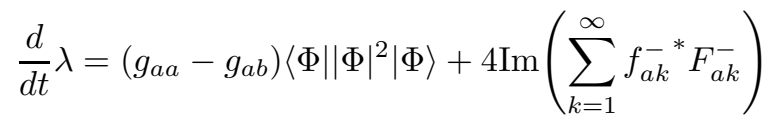

and $\lambda\left(t=0^{+}\right)=0$. The first term in the time evolution in Eq. (56) is the same as the one obtained from the time evolution with a Hamiltonian like (6). The coupling strength (17) was derived by only considering the contribution from the condensate modes, and this coupling constant is the same as the first term in the correct coupling strength in Eq. (57). The second term in Eq. (57) represents a modification of the coupling constant due to the Bogoliubov modes. The atoms in the condensate mode does give the largest contribution to the Hamiltonian (3), but we do not obtain the correct coupling constant by only considering the terms from the condensate modes, because it is the fluctuations of these terms, and not the terms them self, which give rise to phase collapse and squeezing. The fluctuations are of lower order and are comparable to the contributions from



FIG. 1: Modification of the coupling constant due to the Bogoliubov modes. The figure shows the ratio $\gamma$ between the average of the correct coupling constant $d \lambda / d t$ (57) and the average of the single mode value ( $\left(\right.$ ) for $a_{s, a b} / a_{s, a a}=0(+)$, $a_{s, a b} / a_{s, a a}=0.5(\times)$, and $a_{s, a b} / a_{s, a a}=0.93(\triangle)$. The last value corresponds to the value for $\left|F=1, M_{F}= \pm 1\right\rangle$ sodium atoms [30]. In the limit of very weak interactions $(\mu \approx 3 / 2 \omega)$, $\gamma$ approaches the estimate in Eq. (耳), and in the ThomasFermi limit $(\mu \gg \omega)$ it approaches $7 / 10$ as predicted in Ref. [14.

the Bogoliubov modes, and the Bogoliubov modes have to be included. The Hamiltonian (6) is, however, still valid provided that we use the correct coupling constant in Eq. (57).

The presence of the second term in Eq. (57) only changes the coupling constant slightly. Ref. 14 considers the same situation (by a different method) and in the Thomas-Fermi limit $(\mu / \omega \gg 1$, where $\mu$ is the chemical potential) it is shown that the effective coupling constant is approximately $7 / 10$ of the coupling constant in Eq. (7). The functions $f_{a k}^{-}$and $F_{a k}^{-}$appearing in the second term in Eq. (57) comes from the change in the condensate wavefunctions due to the fluctuations in the number of particles in states $a$ and $b$. Outside the the Thomas-Fermi regime the condensate wavefunctions are less affected by the interactions and are closer to the ground state of the harmonic potential which is independent of the number of particles of type $a$ and $b$. We therefore expect that the contribution of the second term becomes smaller if we go away from the Thomas-Fermi limit. This is indeed confirmed by a numerical integration of the equations. In Fig. 11 we show the ratio $\gamma$ between the slopes of linear approximations to the time integrals of Eqs. (57) and (77). In the non-interacting limit $(\mu \approx 3 \omega / 2)$, Eq. (7) gives the correct coupling constant and in the Thomas-Fermi limit $(\mu \gg \omega)$ the correct coupling constant (57) approaches $7 / 10$ of Eq. (7). Note, that this is only true for a time average of the coupling strength. The expression in Eq. (57) have larger oscillation in time.

If we ignore the non-condensed particles, the initial state is a Fock state with $N$ particles of type $a$ in the condensate mode and the vacuum of $\hat{b}$ and $\hat{c}_{k}^{-}$for all $k$. By using the exact relations in Eq. (51) we can calculate 
the shape of the phase collapse

$$
\left\langle J_{x}\right\rangle=\frac{N}{2} \cos ^{N-1}(\lambda) \exp \left(-2 \sum_{k=1}^{\infty}\left|F_{a k}^{-}\right|^{2}\right) .
$$

An example of the phase collapse is shown in Fig. 2, where we show the phase collapse obtained by numerically integrating the equations. The functions $F_{a k}^{-}$perform small oscillations in time and the last exponential in Eq. (58) is of minor importance. The phase collapse arises from the cosine in Eq. (58). The value of $\left\langle J_{x}\right\rangle$ is reduced by a factor $1 / e$ when $\lambda \approx \sqrt{2 /(N-1)}$ and the collapse time is roughly given by

$$
t_{c}=\sqrt{\frac{2}{N-1}} \frac{1}{\gamma \bar{\chi}}
$$

where $\bar{\chi}$ is the average of the coupling constant in Eq. (7). Since $\chi$ is proportional to $1 / N, t_{c}$ scales as $\sqrt{N}$.

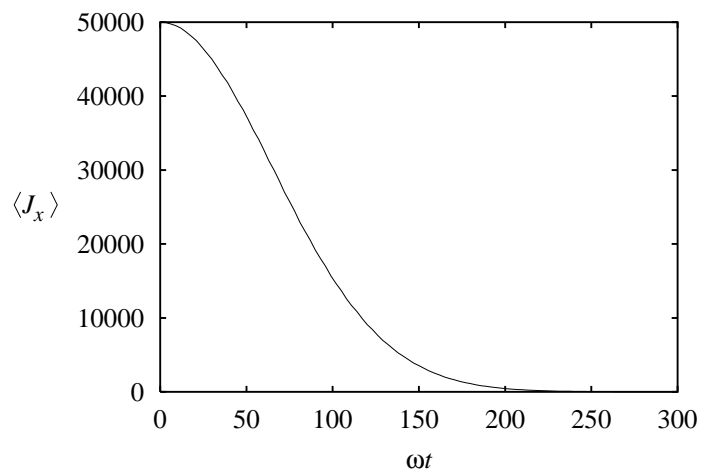

FIG. 2: Collapse of the relative phase between the two components of the condensate. The mean value of the spin $\left\langle J_{x}\right\rangle$ decreases due to the fluctuation in the number of particles in each of the internal states. The parameters are $a_{s, a a} / \sqrt{\hbar / M / \omega}=6 \cdot 10^{-3}, a_{s, b b}=a_{s, a a}=2 a_{s, a b}$, and $N=10^{5}$.

\section{Spin squeezing}

We now turn to the calculation of spin squeezing. The calculation of spin squeezing is more complicated than the calculation of the phase collapse because squeezing is a lower order phenomena. The operators describing the noise are the square of the angular momentum operators and involve the expectation values of the product of four field operators. Such a product is in itself of order $N^{2}$ but due to interferences between different terms of order $N^{2}$ the noise is only of order $N$. Hence to calculate the squeezing we need to be very precise when we calculate the matrix elements. Here we calculate all matrix elements to order $N$ and omit terms of order $\sqrt{N}$ and smaller.

We are interested in estimating the optimal squeezing obtainable with the proposal [4]. Experimentally it is possible to optimize the measurement output by choosing the angle $\theta$ such that the variance is minimized, and by minimizing the variance $\left(\Delta J_{\theta}\right)^{2}$ with respect to $\theta$ we find that the minimum variance is given by

$$
\left(\Delta J_{\theta}\right)_{\min }^{2}=\frac{\left\langle J_{z}^{2}+J_{y}^{2}\right\rangle-\sqrt{\left\langle J_{z}^{2}-J_{y}^{2}\right\rangle^{2}+\left\langle J_{z} J_{y}+J_{y} J_{z}\right\rangle^{2}}}{2}
$$

and that the optimal angle is determined by

$$
\tan (2 \theta)=\frac{\left\langle J_{z} J_{y}+J_{y} J z\right\rangle}{\left\langle J_{z}^{2}-J_{y}^{2}\right\rangle}
$$

To calculate the variance $\left(\Delta J_{\theta}\right)_{\min }^{2}$ it is an advantage to replace $J_{y}$ by the raising and lowering operators $J_{y}=\left(J_{+}-J_{-}\right) / 2 i$, so that, e.g., $\left\langle J_{y}^{2}\right\rangle=\left\langle J_{+} J_{-}\right\rangle / 2-$ $\operatorname{Re}\left(\left\langle J_{+} J_{+}\right\rangle\right) / 2$, where we have used the symmetry to obtain $\left\langle J_{+} J_{-}\right\rangle=\left\langle J_{-} J_{+}\right\rangle$. By expressing the raising and lowering operators in terms of the field operators, using the splitting (9), and introducing the $\hat{\Lambda}_{a}$ operator 18 we obtain

$$
\left\langle J_{+} J_{-}\right\rangle=\left\langle\hat{a}^{\dagger} \hat{a}\right\rangle+\left\langle\hat{a}^{\dagger} \hat{a} \hat{b}^{\dagger} \hat{b}\right\rangle+\bar{N}_{a}\left(\left\langle\hat{\Lambda}_{b}^{\dagger} \circ \hat{\Lambda}_{a}\right\rangle+\left\langle\hat{\Lambda}_{a}^{\dagger} \circ \hat{\Lambda}_{b}\right\rangle\right),
$$

where we have neglected terms of order $\sqrt{\bar{N}_{a}}$. To calculate the first two terms in this expression we use the fact that we have conservation of the number of particles, and that $J_{z}$ commutes with the Hamiltonian because we have no spin changing collisions. From the mean number of particles in the $a$ state $\bar{N}_{a}=\left\langle\hat{\Psi}_{a} \circ \hat{\Psi}_{a}\right\rangle$ we find

$$
\left\langle\hat{a}^{\dagger} \hat{a}\right\rangle=\bar{N}_{a}+O\left(\bar{N}_{a}^{0}\right)
$$

from $\left\langle J_{z}^{2}\right\rangle=N / 4=\left\langle\left(\hat{\Psi}_{a}^{\dagger} \circ \hat{\Psi}_{a}-\bar{N}_{a}\right)^{2}\right\rangle$ we find

$$
\left\langle\left(\hat{a}^{\dagger} \hat{a}\right)^{2}\right\rangle=\frac{N(N+1)}{4}-N\left\langle\hat{\Lambda}_{a}^{\dagger} \circ \hat{\Lambda}_{a}\right\rangle+O\left(\sqrt{\bar{N}_{a}}\right)
$$

and with this expression and $N^{2}=\left\langle\left(\hat{\Psi}_{a}^{\dagger} \circ \hat{\Psi}_{a}+\hat{\Psi}_{b}^{\dagger} \circ \hat{\Psi}_{b}\right)^{2}\right\rangle$ we get

$$
\left\langle\hat{a}^{\dagger} \hat{a} \hat{b}^{\dagger} \hat{b}\right\rangle=\frac{N(N-1)}{4}-N\left\langle\hat{\Lambda}_{a}^{\dagger} \circ \hat{\Lambda}_{a}\right\rangle+O\left(\sqrt{\bar{N}_{a}}\right) .
$$

In the last equation we have made a replacement $\left\langle\delta \hat{\Psi}_{a}^{\dagger} \hat{b} \hat{b}^{\dagger} \delta \hat{\Psi}_{a}\right\rangle=N / 2\left\langle\hat{\Lambda}_{a}^{\dagger} \circ \hat{\Lambda}_{a}\right\rangle+O\left(\sqrt{\bar{N}_{a}}\right)$. This replacement is valid because $\hat{b} \approx \hat{a}$ to lowest order according to Eq. (51). With these equations we can calculate $\left\langle J_{+} J_{-}\right\rangle$ in (62) and by doing a similar calculation for $\left\langle J_{+} J_{+}\right\rangle$we find

$$
\left\langle J_{y}^{2}\right\rangle=\frac{N(N+1)}{8}-\frac{N}{2}\left\langle\hat{\Lambda}_{a}^{\dagger} \circ \hat{\Lambda}_{a}\right\rangle-\frac{1}{2} \operatorname{Re}\left(\left\langle\hat{a}^{\dagger} \hat{a}^{\dagger} \hat{b} \hat{b}\right\rangle\right) .
$$

By similar arguments we can also calculate

$$
\left\langle J_{z} J_{y}+J_{y} J_{z}\right\rangle=2 \operatorname{Im}\left(\left\langle\hat{a}^{\dagger} \hat{a}^{\dagger} \hat{a} \hat{b}\right\rangle\right) .
$$


The matrix elements appearing in Eqs. (66) and (67) cannot be calculated to the desired accuracy by only using Eq. (44), because this equation does not go to high enough accuracy. We need to calculate the matrix elements to order $N$ but in Eq. (44) we are omitting contributions to $\hat{a}$ of order $1 / \sqrt{N}$. The next order correction to $\hat{a}$ gives a contribution of order $N$ when inserted in Eqs. (66) and (67). However we can use the conservation of the number of particles in each of the internal states to find the remaining term in the matrix elements without going to higher order in the calculation of $\hat{a}$. We split the exact operator $\hat{a}$ appearing in Eqs. (66) and (67) into the part that we have calculated so far $\hat{a}_{0}$ and an additional term $\delta \hat{a}$ of order $1 / \sqrt{N}$

$$
\hat{a}=\hat{a}_{0}+\delta \hat{a} .
$$

At $t=0^{+}$we have $\delta \hat{a}=0$ and by using that the number of particles in state $a$ and $\hat{a}_{0}^{\dagger} \hat{a}_{0}$ are independent of time we find

$$
\begin{aligned}
\hat{a}_{0}^{\dagger}(t) \delta \hat{a}(t)+\delta \hat{a}^{\dagger}(t) \hat{a}_{0}(t)= & \hat{\Lambda}_{a}^{\dagger}\left(0^{+}\right) \circ \hat{\Lambda}_{a}\left(0^{+}\right) \\
& -\hat{\Lambda}_{a}^{\dagger}(t) \circ \hat{\Lambda}_{a}(t)
\end{aligned}
$$

Note, that we only need the lowest order contribution of this term, and since $\hat{a}(t) \approx \hat{a}\left(0^{+}\right) \approx \hat{b}\left(0^{+}\right) \approx \hat{b}(t)$ according to Eqs. (43) and (51), we can actually replace $\hat{a}_{0}$ by $\hat{b}_{0}$ which is necessary to complete the calculation below.

With the above relation we are finally able to calculate the relevant matrix elements. Using the splitting in Eq. (68), the initial condition in Eq. (51), the time evolution in Eq. (44), and replacing $\hat{a}\left(t=0^{-}\right)^{\dagger} \hat{a}\left(t=0^{-}\right)$with $N-\hat{\Lambda}_{a}\left(t=0^{-}\right)^{\dagger} \hat{\Lambda}_{a}\left(t=0^{-}\right)=N-2 \hat{\Lambda}_{a}\left(t=0^{+}\right)^{\dagger} \hat{\Lambda}_{a}(t=$ $\left.0^{+}\right)$we obtain

$$
\begin{aligned}
\left\langle J_{y}^{2}\right\rangle= & \frac{N(N+1)}{8} \\
& -\frac{N(N-1)}{8} \cos ^{N}(2 \lambda) \exp \left(-8 \sum_{k=1}^{\infty}\left|F_{a k}^{-}\right|^{2}\right)
\end{aligned}
$$

and

$$
\begin{aligned}
\left\langle J_{z} J_{y}+J_{y} J_{z}\right\rangle=- & \frac{N(N-1)}{4} \cos ^{N}(\lambda) \sin (\lambda) \\
& \times \exp \left(-2 \sum_{k=1}^{\infty}\left|F_{a k}^{-}\right|^{2}\right) .
\end{aligned}
$$

By inserting these two expressions into Eq. (60) we can find the minimum variance and with the expression for $\left\langle J_{x}\right\rangle$ in Eq. (58) we may find the squeezing parameter $\xi_{\theta}^{2}$.

Note that we have not specified anything about the initial state of the + modes. Within the approximations used here, the results (70) and (71) only involves expectation values of operators for the - modes and the squeezing is independent of the state of the + modes. If the condensate is at a non-zero temperature before



FIG. 3: Time Evolution of the squeezing calculated by a numerical integration (full line) and from the approximate Hamiltonian (6) (dashed curve). The parameters are the same as in Fig. 目.

the pulse, it can be described by quasi-particle excitations, and these excitation are transfered into excitation in the + modes after the pulse. The - modes are always in the vacuum state independent of temperature, and the squeezing calculated here is therefore also independent of temperature. Another nice property of Eqs. (70) and (71) is that they only involve the functions $F_{a k}^{-}$ and not the number of non-condensed particles. Because the functions $F_{a k}^{-}$vanish for states with non-zero orbital angular momentum, according to Eq. (40), it is sufficient to determine the evolution of the Bogoliubov modes with vanishing orbital angular momentum and this simplifies a numerical treatment of the system significantly.

In Fig. 3 we show the evolution of spin squeezing with the same parameters as in Fig. 2. In the figure we also show the prediction of the Hamiltonian (6) with a coupling constant $\chi$ equal to the slope of a linear approximation to $\lambda$. The two curves are in reasonably good agreement with each other. The deviation between the two curves is caused by the exponentials of the $F_{a k}^{-}$functions in Eqs. (70) and (71), i.e., by the dependence of the wavefunction on the number of particles. After the resonant pulse the wavefunction is no longer in equilibrium because the repulsion of the atoms is suddenly reduced, and the size of the atomic cloud will oscillate in time. The oscillations of the wavefunction depend on the number of atoms in each of the internal state, and because the state of the system is distributed on states with different number of atoms in each internal state, there is an uncertainty in the wavefunction which introduces noise and reduces the squeezing. After the completion of a full oscillation ( $\omega t \approx 4,9,13$, and 18$)$, the wavefunction has approximately its initial form independent of the number of atoms in each internal state $\left(F_{a k}^{-} \approx 0\right)$, and the results of the Hamiltonian (6) are in very good agreement with the numerical results. The result in Fig. 3 is very similar to the result obtained with the same parameters but a different approximate method in Ref. [i] , and our result thus supports the conclusions reached in that paper. 


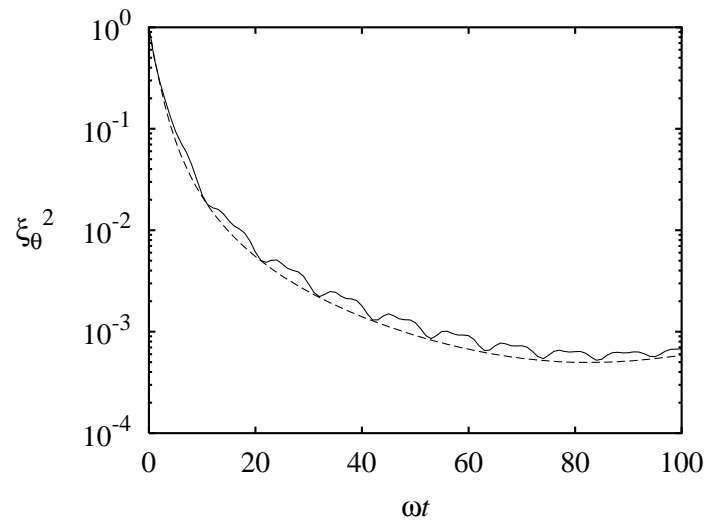

FIG. 4: Squeezing between the two $\left|F=1, M_{F}= \pm 1\right\rangle$ hyperfine states in sodium. The parameters are the same as in Fig. 2 except $a_{s, a b} / a_{s, a a}=0.93$. The full curve is the result of a numerical simulation and the dashed curve is from the Hamiltonian (6).

The difference in scattering length used in Fig. 3 is an exaggeration of the realistic parameters. If the experiment is performed with the two hyperfine states $\left|F=1, M_{F}= \pm 1\right\rangle$ in sodium representing the internal states $a$ and $b$ as proposed in [A], the ratio between the scattering lengths is $a_{s, a b} / a_{s, a a} \approx 0.93$ 30. In Fig. A we show the squeezing produced with this value of the ratio. Because the scattering lengths are very close, the oscillations of the wavefunction are much smaller, and the numerical curve and the result from Eq. (16) are in better agreement.

To create an entangled state the coupling constant $\chi$ in the Hamiltonian (6) should be non-zero. So far we have obtained a non-zero coupling by having a difference in the the scattering lengths $a_{s, a a}+a_{s, b b} \neq 2 a_{s, a b}$. From the approximate coupling constant in Eq. (7) we see that $\chi$ is also non-zero if the $a$ and the $b$ atoms occupy two different regions in space, even if all scattering lengths are identical. In Ref. [9] it was proposed to produce spin squeezing by replacing the two internal states $a$ and $b$ with two different momentum states which are created by a Bragg pulse shortly after the trap has been turned off, i.e., exactly the same setup as used in 31. This proposal has the advantage that the squeezing is now between atoms in the same internal state, and the squeezing is therefore better shielded from phase decoherence caused for instance by fluctuating magnetic fields. Also, this proposal could be used for rubidium atoms where the scheme which has been studied so far is not applicable because the scattering lengths are almost identical.

To investigate this situation we assume that the time it takes the two momentum states to separate is very short so that we can neglect the interaction during this separation process. In this case there is no interaction between the $a$ and the $b$ components and we can describe this situation by setting $a_{s, a b}=0$ and $V_{a}=V_{b}=0$ after the pulse. In Fig. 5 we show the result of such a simulation with parameters similar to the parameters in Ref.
31. The calculation shows that strong squeezing can be produced with this proposal, but the agreement with the Hamiltonian (6) is not as good in this case because of the exponentials in Eqs. (70) and (71).

\section{CONCLUSION}

In this paper we have analysed the scheme proposed in Ref. [4] by describing the system with a numberconserving Bogoliubov theory. The developed theory is a consistent expansion in the ratio between non-condensed and condensed particles, and the validity of the calculations can be investigated in a given experimental configuration.

The results obtained in this paper show that strong squeezing can indeed be produced by this method, but the theory is not able to determine the precise limit of the obtainable squeezing. The results of this paper agree with the result of the simplified Hamiltonian (6) which predict a reduction of the squeezing parameter by a factor of approximately $N^{-2 / 3}$, but we are ignoring terms of order $\sqrt{\delta N / N}$. From this we conclude that the obtainable squeezing is at least of order $\sqrt{\delta N / N}$. The obtained results are independent of the temperature of the condensate, and the only effect of a non-vanishing temperature is that the approximations break down a little earlier.

\section{Acknowledgments}

I am grateful to Klaus Mølmer and Uffe V. Poulsen for useful discussions and comments on the manuscript.

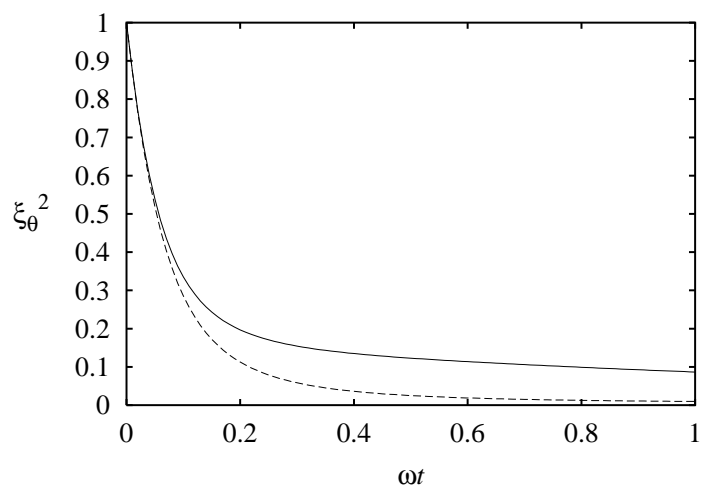

FIG. 5: Squeezing created by a Bragg pulse as proposed in Ref. 9]. At time $t=0$ the trap is turned off and two components are separated by a Bragg pulse. The full line is the result of a numerical integration and the dashed curve is the prediction from the Hamiltonian (6). The parameters are $a_{s, a a} / \sqrt{\hbar / M / \omega}=6 \cdot 10^{-4}, a_{s, b b}=a_{s, a a}, a_{s, a b}=0$, and $N=1.7 \cdot 10^{6}$. These parameters are chosen such that they are similar to the parameters in Ref. 31]. The ratio $a_{s, a a} / \sqrt{\hbar / M / \omega}=6 \cdot 10^{-4}$ corresponds to sodium atoms in a trap with trapping frequency $\omega=2 \pi \cdot 19 \mathrm{~Hz}$ before the trap is turned off. 
This work was supported by the Danish National Research Foundation through the Quantum Optics Center
(QUANTOP) in Århus.
[1] M. H. Anderson, J. R. Ensher, M. R. Matthews, C. E. Wieman, and E. A. Cornell, Science 269, 198 (1995).

[2] K. B. Davis, M.-O. Mewes, M. R. Andrews, N. J. van Druten, D. S. Durfee, D. M. Kurn, and W. Ketterle, Phys. Rev. Lett. 75, 3969 (1995).

[3] A. S. Parkins and D. F. Walls, Physics Reports 303, 1 (1998).

[4] A. Sørensen, L.-M. Duan, J. I. Cirac, and P. Zoller, Nature 409, 63 (2001).

[5] L.-M. Duan, A. Sørensen, J. I. Cirac, and P. Zoller, Phys. Rev. Lett. 85, 3991 (2000).

[6] H. Pu and P. Meystre, Phys. Rev. Lett. 85, 3987 (2000).

[7] S. Raghavan, H. Pu, P. Meystre, and N. Bigelow, Optics Comm. 188, 149 (2001).

[8] U. V. Poulsen and K. Mølmer, Phys. Rev. A 64, 013616 (2001).

[9] U. V. Poulsen and K. Mølmer, cond-mat/0109048.

[10] K. Helmerson and L. You, Phys. Rev. Lett. 87, 170402 (2001).

[11] L.-M. Duan, J. I. Cirac, and P. Zoller, quant-ph/0107055.

[12] D. J. Wineland, J. J. Bollinger, W. M. Itano, and D. J. Heinzen, Phys. Rev. A 50, 67 (1994).

[13] M. Lewenstein and L. You, Phys. Rev. Lett. 77, 3489 (1996).

[14] A. Sinatra and Y. Castin, Eur. Phys. J. D 8, 319 (2000).

[15] Y. Castin and R. Dum, Phys. Rev. A 57, 3008 (1998).

[16] J. L. Sørensen, J. Hald, and E. S. Polzik, Phys. Rev. Lett. 80, 3487 (1998).

[17] A. Kuzmich, L. Mandel, and N. P. Bigelow, Phys. Rev. Lett. 85, 1594 (2000).

[18] C. Orzel, A. K. Tuchman, M. L. Fenselau, M. Yasuda, and M. A. Kasevich, Science 291, 2386 (2001).
[19] B. Julsgaard, A. Kozhekin, and E. S. Polzik, Nature 413, 400 (2001).

[20] G. Santarelli, Ph. Laurent, P. Lemonde, A. Clairon, A. G. Mann, S. Chang, A. N. Luiten, and C. Salomon, Phys. Rev. Lett. 82, 4619 (1999).

[21] A. S. Sørensen and K. Mølmer, Phys. Rev. Lett. 86, 4431 (2001).

[22] M. Kitagawa and M. Ueda, Phys. Rev. A. 47, 5138 (1993).

[23] A. S. Sørensen and K. Mølmer, unpublished.

[24] C. W. Gardiner, Phys. Rev. A 56, 1414 (1997).

[25] M. Girardeau and R. Arnowitt, Phys. Rev. 113, 755 (1959); M. D. Girardeau, Phys. Rev. A 58, 775 (1998).

[26] F. Dalfovo, S. Giorgini, M. Guilleumas, L. Pitaevskii and S. Stringari, Phys. Rev. A 56, 3840 (1997).

[27] Compared to the derivation in Ref. 15] we have replaced $\hat{N}_{a}$ appearing in the definition of $\hat{\Lambda}_{a}^{\mathrm{ex}}(18)$ with the number $\bar{N}_{a}$. In our situation the number of particles in the $a$ state is not a fixed number, and the arguments leading to Eq. (19) are easier to perform with a number $\bar{N}_{a}$.

[28] A. Sørensen and K. Mølmer, Phys. Rev. A 62, 022311 (2000).

[29] Actually, $\hat{b}$ and $\delta \hat{\Psi}_{b}$ are not uniquely defined before the pulse because there is no $b$ component of the condensate. The operators appearing in Eqs. (51) and (54) are defined by using the condensate modefunction for the $a$ particles.

[30] C. Williams, private communications.

[31] J. Denslag, J. E. Simsarian, D. L. Feder, C. W. Clark, L. A. Collins, J. Cobizolles, L. Deng, E. W. Hagley, K. Helmerson, W. P. Reinhardt, S. L. Rolston, B. I. Schneider, and W. D. Phillips, Science 287, 97 (2000). 